\title{
Dust evolution in the star forming turbulent interstellar medium
}

\author{
François Boulanger ${ }^{1}$ \\ ${ }^{1}$ Institut d'Astrophysique Spatiale, Université Paris-Sud 11 and CNRS (UMR 8617), F-91405 \\ Orsay \\ boulanger@ias.u-psud.fr
}

\begin{abstract}
Understanding interstellar dust evolution is a major challenge underlying the interpretation of Spitzer observations of interstellar clouds, star forming regions and galaxies. I illustrate on-going work along two directions. I outline the potential impact of interstellar turbulence on the abundance of small dust particles in the diffuse interstellar medium and translucent sections of molecular clouds. I present results from an analysis of ISO and Spitzer observations of the central part of 30 Doradus, looking for dust evolution related to the radiative and dynamical impact of the R136 super star cluster on its parent molecular cloud.
\end{abstract}

Keywords. ISM:Structure, ISM: Evolution, Dust, Stars: Formation

\section{Introduction}

Interstellar dust has only a distant kinship with star dust. Its composition, abundance and size distribution reflects the action of interstellar processes that contribute to break and re-build grains over time-scales much shorter than the renewal time scale by stellar ejecta. If there is a wide consensus on this statement, observations only provide fragmented evidence of dust evolution and we have yet to understand where in interstellar space and how dust evolution occurs?

This research field is relevant to the symposium topic in several ways. The impact of dust evolution on the interstellar medium (ISM) chemical and physical state and on star formation are wide-ranging and still largely unexplored. On the one hand, dust evolution underlies the interpretation of infrared observations used to trace the condensation of interstellar matter from the diffuse interstellar medium to pre-stellar cores and estimate star formation rates in galaxies. On the other hand, interstellar dust studies need to be set in the general framework of the ISM dynamical evolution including the feedback of newly born stars on their environment.

The physical processes acting on dust leave specific signatures on the dust size distribution and optical properties which in turn affects the dust spectral energy distribution (SED). With spectral bands measuring specifically the emission features from PAHs, the mid-IR emission from stochastically heated very small grains (VSGs) and the far-IR emission from large grains, the Spitzer Space Telescope imaging instruments IRAC and MIPS are particularly well suited to map the relative abundance of dust in these three size bins, while the IRS spectrometer can provide spectroscopic insight for PAHs and VSGs.

The Galactic and nearby galaxies Spitzer surveys and dedicated observing programs open many new perspectives to study dust evolution from small scales in the nearby interstellar medium to Galactic scales. The analysis of these data has just started. Only very few secure results have been obtained. Most of this research is ahead of us. For this paper, I chose to illustrate on-going work along two directions relevant to the symposium 
topic. First, we outline the potential impact of interstellar turbulence on the dust mass fraction in PAHs and VSGs. Second, we present results from an analysis of dust emission around the R136 super star cluster at the center of 30 Doradus.

\section{Small dust particles in the turbulent interstellar medium}

A significant fraction of the dust mass is in PAHs and VSGs (e.g. Li \& Draine 2001) but their abundance is observed to vary across the diffuse interstellar medium and at the surfaces of molecular clouds (e.g. Miville Deschênes et al. 2002). The processes responsible for these abundance variations have yet to be identified.

UV and also X-ray photons that permeate the diffuse ISM and the translucent sections of molecular clouds can alter dust by photo-physical and chemical processing. But far from massive star forming regions gas-grain and grain-grain collisions are thought to be the dominant evolutionary processes. The degree and nature of the processing depends on the rate and the energy of these interactions, both of which are related to the density structure and dynamics of the ISM.

Grain sputtering and shattering in fast shock waves are thought to dominate the overall evolution of interstellar dust in the warm interstellar medium (Jones et al. 1996). Metals depletions observed to be lower in the warm interstellar medium than in clouds and changes in the gas-to-dust mass ratio (Bot et al. 2004, Borkowski et al. 2006, Stanimirovic et al. 2005) are evidence for return of dust mass into the gas phase that could be explained by shock destruction.

Turbulence within the cold interstellar medium generates shocks and vortices which might affect the dust evolution more frequently and more deeply on average than fast shocks. In turbulent clouds, the outcome from grain-grain collisions depends on grains relative velocities. Below some size dependent critical velocity, grains are expected to stick to each other (Chokshi et al. 1993), while above some threshold collision energy, grain shattering produces smaller fragments (Jones et al. 1996). Grain relative velocities are set by turbulent motions and depend on the grain size (i.e. their mass to surface ratio) and their charge state through the coupling with the magnetic field. Yan et al. (2004) have calculated the relative grain motions arising from magneto hydrodynamic turbulence as a function of ISM physical conditions. They find that the largest velocities are always those of the large grains but VSGs can also be efficiently decoupled from gas motions by large velocity gradients at small scales, known to exist in turbulence and not included in the Yan et al. (2004) work (Falgarone and Puget 1995, Falgarone this volume). Where this occurs, PAHs and VSGs mutually coagulate faster than they stick on large grains.

The gas turbulent velocity which acts as a scaling factor on the grain relative velocities and the gas density are the main physical parameters determining the collision rates and collisions outcome. PAHs and VSGs abundance is locally set by the rates at which grain-grain collisions lead to their production by grain shattering, their growth in size by mutual coagulation or their disappearance when they stick on large grains. Time-scales are uncertain - they depend on empirical factors that await to be determined through observational constraints - but diffuse clouds and the translucent sections of molecular clouds span the critical range of physical conditions where we expect to observe the interplay between dust evolution and the clouds density and velocity structure. Correlation between observed signatures of small dust evolution and physical parameters are presently the missing clues to characterize the evolutionary processes acting on PAHs and VSGs abundance. 


\section{Dust evolution near the 30 Doradus super star cluster}

Spitzer observations are opening a new perspective on massive star formation from the Galaxy to distant galaxies. Mid-IR Spectra of star bursts like those of massive star forming regions are a combination of PAH bands and continuum from stochastically heated VSGs. The key questions raised in many studies is how can the spectral energy distribution be used to investigate the nature and the evolutionary stage of extra galactic IR sources and to estimate star formation rates? To answer these questions it is necessary to understand massive stars impact on dust in star forming regions. PAH bands are widely used as a spectroscopic signature to distinguish between infrared galaxies powered by star bursts rather than an active galactic nucleus. But PAH bands are also weak and even absent in low metallicity star forming galaxies (e.g. Engelbracht et al. 2005, Madden et al. 2006). Does this reflect PAHs destruction in H II regions (e.g. Giard et al. 1994) or a metallicity effect on dust composition?

\subsection{Interstellar matter near the R136 Super Star Cluster}

At the center of 30 Doradus, the R136 star cluster is the only super star cluster sufficiently close to have a detailed view at the cluster impact on the surrounding interstellar medium and dust. Interstellar matter in 30 Doradus has been the topic of many papers over the last decades. There are two CO clouds at the center of the nebula to the North and South West (SW) of R136. These clouds have the same radial velocity and are possibly two pieces of a single parent cloud where the cluster was born.

Spectacular IRAC images of 30 Doradus have been released early in the Spitzer mission. We present results from an analysis of these images on a $3^{\prime} \times 3^{\prime}$ field around the R136 cluster covered by an Infrared Space Observatory (ISO) spectroscopic observation. This field encompasses the ionization front and the photo-dissociation region (PDR) at the surface of the SW molecular cloud. A complete account of this work is presented in Boulanger et al. (2007).

The interaction of R136 with its parent cloud may be understood within the theoretical framework set by McKee et al. (1984) to describe the expansion of photo-ionized wind bubbles in a clumpy medium. Stellar winds have carved out a cavity within the cloud. The winds have burst out of the molecular clouds in many directions and thereafter expanded out faster in the tenuous surrounding medium powering the outer shells seen in wider images of the nebula. In the direction of the CO clouds, the expansion is still occurring within the clouds. The H II filaments observed at the edge of the two clouds delineate the wind-swept shell. Taking the molecular cloud velocity as a reference, in direction of R136 the shell is observed to be moving outwards at a velocity of $10-20 \mathrm{~km} \mathrm{~s}^{-1}$ (Peck et al. 1997).

The CO emission has been interpreted as arising form the shielded interiors of dense clumps with a small (5-10\%) surface filling factor (Poglitsch et al. 1995, Pak et al. 1997). These two papers do not set a clear constrain on whether the inter-clump gas is neutral or ionized. At faint levels the $\operatorname{Br} \gamma$ emission extends beyond the shell. This faint emission could arise from the outer surface of the molecular cloud but it may also indicate that the R136 Lyman continuum photons propagate into the molecular cloud, ionizing the clump surfaces and the inter-clump gas. In a clumpy medium one does expect the ionization radiation to propagate ahead of the wind swept shell in the lower density gas (McKee et al. 1984). The numerical simulations presented in this volume support and illustrate this view. Within this picture, the expansion of the wind swept shell does not drive a large scale shock in the molecular cloud but ablates matter from the clumps into turbulent 


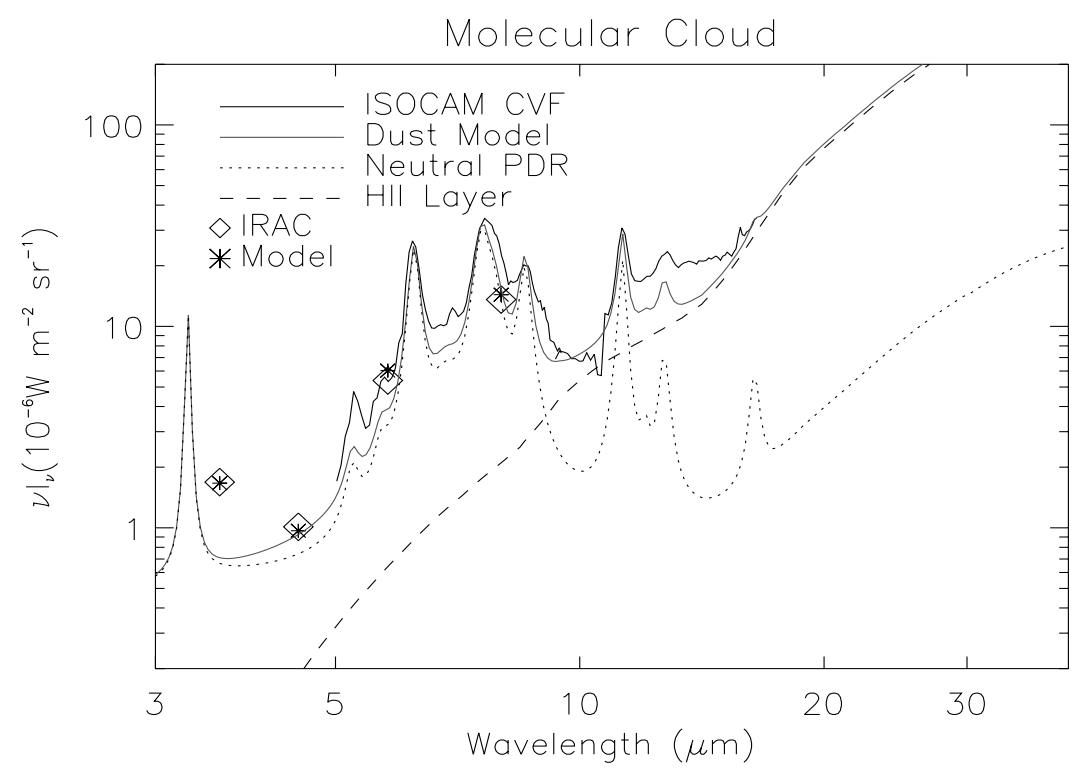

Figure 1. Mid-IR spectrum of the 30 Doradus molecular cloud with proposed decomposition into emission from the ionized and neutral layers of photo-evaporating clumps.

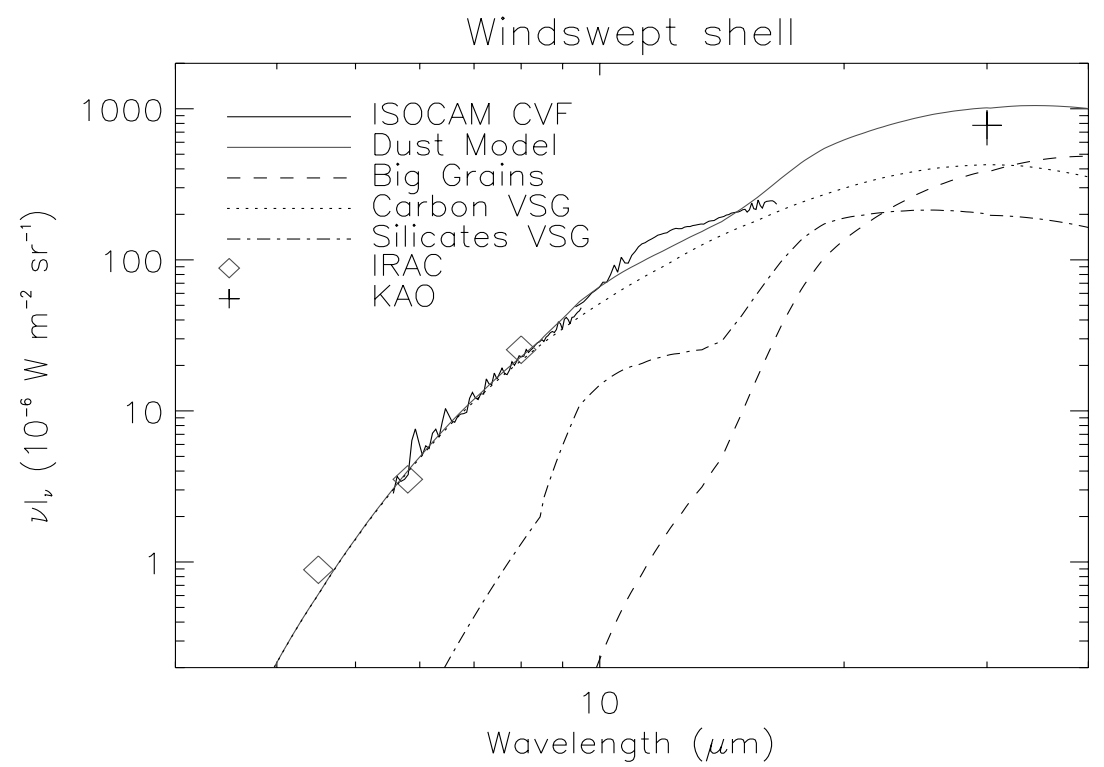

Figure 2. Mid-IR spectrum of ionized shell swept by winds from the 30 Doradus R136 super star cluster.

flows (Nakamura et al. 2006). Dust evolution could also here be driven by the turbulent gas dynamics.

\subsection{Dust spectral energy distribution and ISM structure}

The shell geometry permits to spatially separate the infrared emission from the ionized wind-swept shell from that arising from the molecular cloud. The SEDs derived from this 


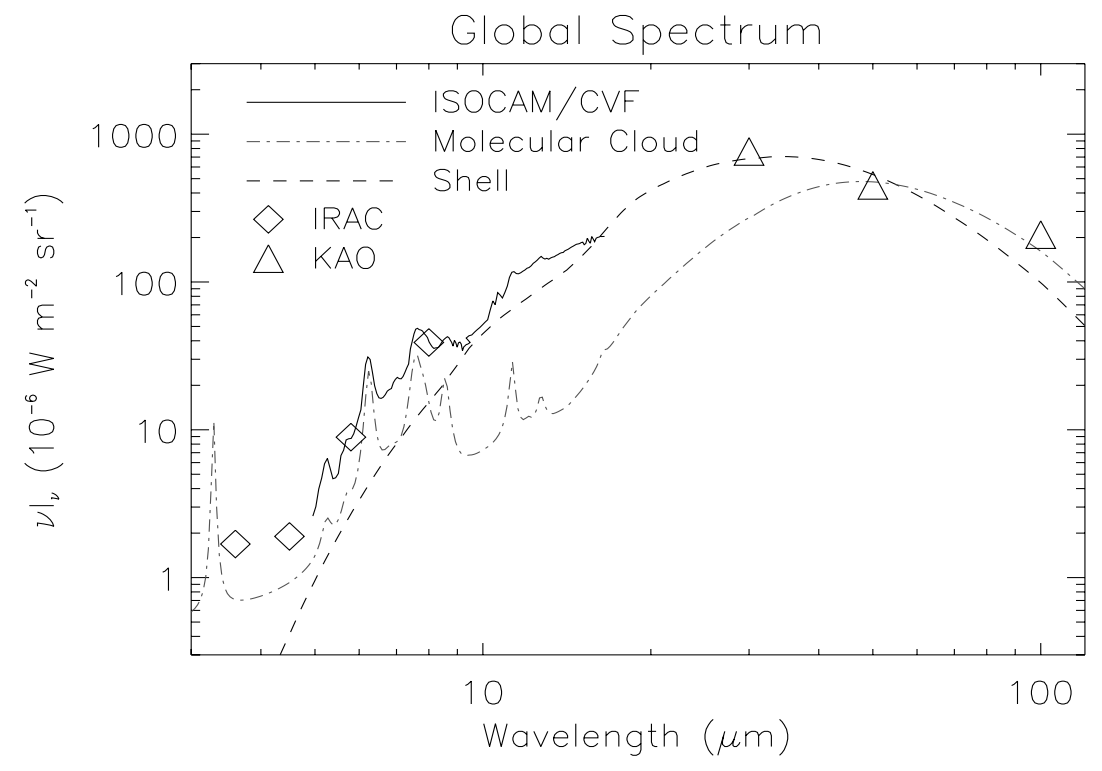

Figure 3. Global spectrum at the far-IR brightness peak to the West of R136 integrated over the 1' aperture of Kuiper Airborne Observatory observations.

correlation analysis are presented in Figs. 1 and 2. In each figure the data are compared to model calculations taking into account differences in the stellar radiation field through the $\mathrm{HII} / \mathrm{H}_{2}$ transition.

The fit of the molecular cloud spectrum builds on our view at at its structure. The far-UV radiation field at the surface of the 30 Doradus molecular clumps is comparable to that at the surface of the archetype PDR NGC 7023. But the 30 Doradus molecular cloud spectrum differs from that of NGC 7023 in two significant ways: the PAH bands are weaker by a factor 6 and the continuum is stronger. In the model, the 30 Doradus $\mathrm{PAH}$ emission is weak because it is arising from clumps which only fill a small fraction of the telescope beam and the mid-IR continuum represents emission from PAH-free ionized gas evaporating off the clumps. Modeling of the wind-swept shell SED yields insight on dust evolution. The smallest carbon particles including PAHs are destroyed. The mid-IR continuum comes from carbon VSGs with sizes larger than 5000 atoms. The absence of the $9 \mu \mathrm{m}$ silicate feature in emission sets a lower limit on the small size cut-off of the silicates size distribution a factor 4 larger.

The 30 Doradus SEDs are keys to the interpretation of infrared observations of massive star forming regions and galaxies. The relative contributions of the molecular cloud and the wind-swept shell to the 30 Doradus emission varies with wavelength (Fig. 3). Different infrared wavelengths highlight the emission from different components of star forming regions. The molecular cloud contribution is dominant at short and long wavelengths while emission from dust in HII gas dominates in the mid-IR. The relative scaling between the two contributions depends on the ISM structure. For example, the PAH emission bands contrast depends on the degree of clumping of the molecular matter. In massive star forming regions like in turbulent clouds away from massive stars (Sect. 2), the dust emission spectrum reflects the interplay between dust evolution and the ISM structure. Conclusions on dust composition and evolution may be reached only within an 
understanding of the radiative and dynamical impact of massive stars on the interstellar medium structure.

\section{Acknowledgements}

I thank Edith Falgarone for sharing her insight on interstellar turbulence and many discussions on its potential impact on dust evolution.

\section{References}

Borkowski, K. J., Williams, B. J. \& Reynolds, S. P., et al. 2006, ApJ 652, 1259

Bot, C., Boulanger, F. Bot, C., Lagache, G., Cambrésy, L. \& Egret, D. 2004, A $\mho A$ 423, 567

Boulanger, F., Rubio, M., Bot, C. \& Viallefond, F. 2007, to appear in $A \mathscr{E} A$

Chokshi, A., Tielens, A. G. G. M. \& Hollenbach, D. 1993 ApJ 407, 806

Engelbracht, C. W., Gordon, K. D., Rieke, G. H., Werner, M. W., Dale, D. A. \& Latter, W. B. 2005, ApJ 628, L29

Falgarone, E. \& Puget, J. L. 1995 A $\& A$ A 293, 840

Giard, M., Bernard, J. P., Lacombe, F., et al. 1994, A\& A 291, 239

Jones, A., P., Tielens, A. G. G. M. \& Hollenbach, D. J. 1996, ApJ 469, 740

Li, A. \& Draine, B. T. 2001 ApJ 554, 778

Madden S. C., Galliano, F., Jones, A. P. \& Sauvage, M. 2006, A\& A 446, 877

McKee, C. F., Van Buren, D. \& Lazareff, B. 1984, ApJ 278, L115

Miville-Deschênes, M.-A., Boulanger, F., Joncas, G. \& Falgarone, E. 2002, A\&A 381, 209

Nakamura, F., McKee, C. F., Klein, R. I. \& Fisher, R. T. 2006, ApJS 164, 477

Pak, S., Jaffe, D. T., van Dishoeck, E. F., Johansson, L. E. B. \& Booth, R. S. 1998, ApJ 498, 735

Peck, A. B., Goss, W. M., Dickel, H. R., et al. 1997, ApJ 486, 329

Poglitsch, A., Krabbe, A., Madden, S. C., et al. 1995, ApJ 454, 293

Stanimirovic, S., Bolatto, A. \& Sandstrom, K., et al. 2005, ApJ 632, L103

Yan, H., Lazarian, A. \& Draine, B. T. 2004, ApJ 616, 895

\section{Discussion}

PADOAN: Is there any reason why large increase in dust/gas ratio with density should not occur in our galaxy, while they occur in the Magellanic Clouds? We have found evidence of that in Taurus (Padoan et al. 2006).

BOUlANGER: Observed changes in metal depletions show that dust to gas ratio also varies in the Milky Way from the Warm tenuous gas to cold dense gas. But the amplitude of the variation may be smaller than in the SMC. The variation in dust to gas ratio is expected to depend on the ratio between energy injection by stars which trigger dust destruction and mass in dense clouds where dust rebuilding occurs.

Goldman: What processes are dominant in dust destruction: mechanical or radiative? and what are typical time scales for destruction?

BOUlANGER: Both mechanical (e.g. shattering in grain-grain collisions) and radiative (e.g., small grain explosion following multiple ionization, photo evaporation) are probably at work but analysis of more data is necessary to characterize destruction processes and identify relevant physical parameters (e.g., radiation field intensity and hardness, $\mathrm{x}$-ray flux, turbulence amplitude, shocks) for dust evolution. This is a main topic to be addressed with the Spitzer LMC and SMC surveys. 